\title{
A Universal Model for the future : The Quanta factor theory of the mind
}

\author{
Rohan Pillai \\ IBDP Psychology Faculty, Fazlani L'Academie Globale, Mumbai. \\ E-mail - rohanpillai@hotmail.com
}

\begin{abstract}
Providing an empirical conceptualization of the human mind specifically consciousness, can be considered as the greatest challenge for psychology in the future. For some time now mathematicians and physicists have been attempting to explain consciousness with the theories and assumption of their domains. But no matter how elegant a natural science proposal maybe, it will not be considered valid unless it envisions and elaborates precisely what it translates to in behavioral terms. Having said that, one tenet of natural science that appealed to me was that there cannot be two separate explanations of the same phenomenon. This was the basis for an intriguing race towards the unification of theories in physics and the search for a universal theory of everything. This situation motivated me to attempt to consider a universal model of human behavior, both covert and overt, and this attempt has materialized onto the creation of "The Quanta Factor Theory".
\end{abstract}

Key words: mind, consciousness, quanta factor theory, unification.

(Paper received $-10^{\text {th }}$ June 2016, Review completed $-22^{\text {nd }}$ June 2016, Accepted $-23^{\text {rd }}$ June 2016)

\section{INTRODUCTION}

In this paper, I present a brief introduction of that theoretical model namely 'The Quanta Factor Theory of the Human Mind', that envisions a number of constructs and functional components that are proposed to be sufficient to explain all facets of human behavior and mental processes. At the present time, in its current form the theory is an elaborate tentative hypothesis wherein most of its components are hypothetical and their implications are verified by reinterpreting results of well known psychological experiments in quanta factor theoretical terms. The detailed descriptions of concepts and their implications are explained in my book by the same name, however for the purpose of this paper I have presented a brief explanation of all the significant components of the theory and listed a few quanta factor theoretical conceptualization of some mental processes and behaviors.

\section{THE QUANTA FACTOR THEORY IN BRIEF}

The most basic explanation of the theory would be this:

At every saccade as human beings encounter sensory information of all modalities by both internal and external sense organs that information is broken down in its least divisible units and is merged with the latent affect within the individual and this information in stored in the form of hypothetical constructs called 'quanta factors'. All the quanta factors made during one saccade are stored in one frame. And with each passing saccade a new frame is made and human experience is thus cataloged and stored permanently in frames. Then during the creation of every new frame due to (but not limited to) resonance, the wave of personal space and the guided assimilation based reality, information held in previously stored frames is made available at the currently forming frame and based on certain rules and probabilities some 
of this information is also merged in the latest frame along with the new incoming information thus influencing our current experience. These constructs and processes are proposed to be sufficient to explain every facet of human behavior and mental processes. This is a very basic explanation of the theory.

I shall now elaborate on the concepts and functioning and a few implications of the quanta factor theory in concise. To briefly explain the theory I am dividing the concepts in the theory in two parts namely the structural components and the functional components. Structural components are those components of the theory that are the hypothetical constructs and the manner in which they are proposed to be placed. The functional components are those concepts envisioned to maintain a relationship between the structural components and allow the functioning of the theory.

\section{STRUCTURAL COMPONENTS OF THE THEORY}

\section{The Quanta Factor}

The theory starts with the proposal of a hypothetical construct called a Quanta Factor. This construct is proposed to be a unit of energy that holds information. One Quanta Factor is made up of two parts. One is the Least Divisible Unit of Latent Affect (LDU of LA) and the other is the Least Divisible Unit of Sensory Information (LDU of SI). LDU of SI is to be understood as the smallest individually perceivable unit of sensory information in all sensory modalities. For example a single wavelength, intensity or edge in the visual field at a given moment for the visual sense, a single frequency, pitch or timber perceived at a given moment for the auditory sense and so on for all senses. If you are looking at a plate of food and you can smell the food then every color, edge, boundary etc that you perceive in front of you it is proposed, is being broken down into LDU's of visual sensory information (VSI). At the same time all the smells that can be perceived as distinct by your olfactory sense are being cataloged as the LDU's of olfactory sensory information (OSI). This break up of sensory information is envisioned for all sense modalities. And this process creates one half of all the Quanta Factors that are being created at that moment, namely the LDU's of SI. The second part of a Quanta Factor is the Least Divisible Unit of Latent Affect. Latent Affect in the QFT is hypothesized to be primordial sensations inherent within and naturally elicited and experienced involuntarily by organisms. It is to be understood as all the labeled or unlabeled inner feelings and sensations that are experienced by human beings. In the QFT a latent affect is proposed to be experienced continuously. The LDU of LA can never be labeled as any particular emotion or feeling.

\section{Figure 1 - A single quanta factor}

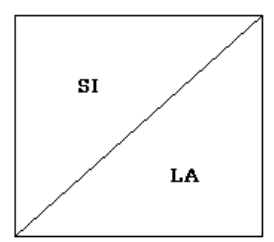

\section{Frames}

Thus the LDU's of SI and LA of a given moment merge and form Quanta Factors of that moment. They are cataloged and are proposed to have a permanent existence. These QF's are stored in what the QFT calls a 'frame' that pertains to that moment. A frame is a collection of QF's that are created in one single moment. It is the repository of all the LDU's of SI and the LDU of LA of that moment. At any given moment only one LDU of LA can be experienced. Thus a frame may be made up of several LDU's of SI but shall always have only one LDU's of LA. All the LDU's of SI of all modalities experienced in one saccade merge with a single LDU of LA that is being experienced by the individual in that saccade. One must understand that though the latent affect in one frame is the same, different LDU's of SI of various modalities are merged separately with that latent affect creating separate individual Quanta Factors as seen below. QF's are labeled based on the sensory modalities they are made up of.

And one frame with the QF's created by the merging of LDU's of five sensory modalities can be visualized as: 


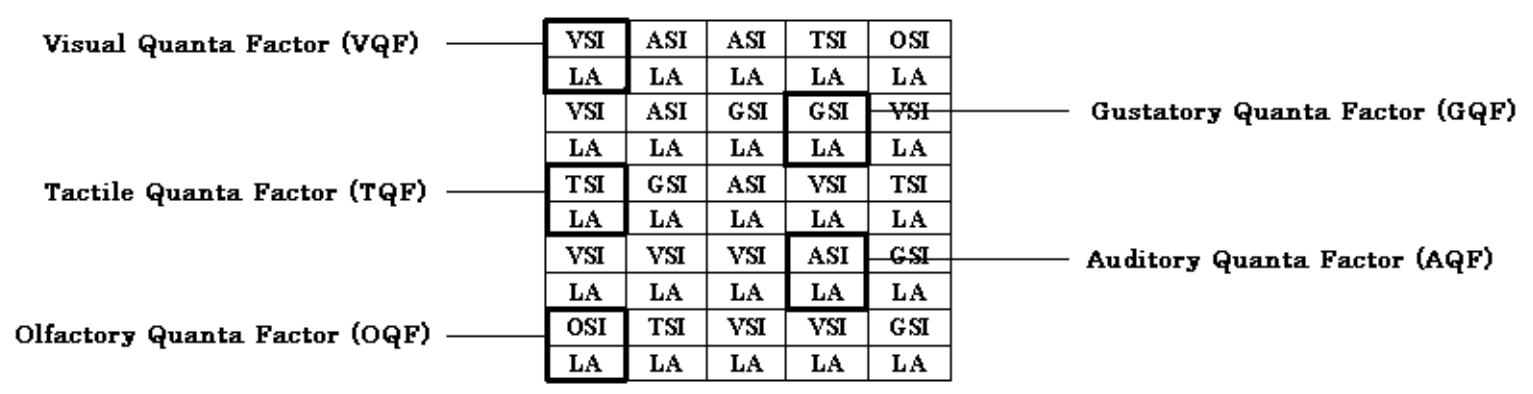

Figure 2 - One frame with QF's of different modalities merging with the same Latent Affect separately.

\section{Space and Time of Consciousness}

Quanta Factors are considered as the building blocks of consciousness in the QFT. And the continuous experience of consciousness is proposed to be the result of the constant creation of frames. The QFT has set the duration of the creation of frames as one frame per saccade. That roughly equates to three frames per second. These frames are proposed to be stored in personal space. It is envisioned that frames are cataloged as an array. The frame which is being currently created is called the frame alpha or f $\alpha$. An individual's collection of frames is known as the individual's personal space. Thus each one of us is proposed to have a personal space of our own which is the collection of all the QF's that we have ever created. In other word it is a repository of every conscious, subconscious and unconsciousness experience converted in the form of Quanta Factors.

Thus a segment of personal space could be imagined as thus:

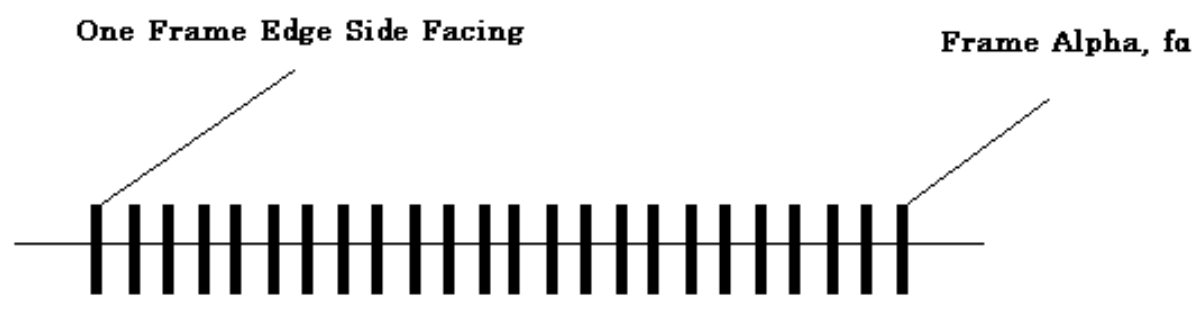

Array of Frames in Personal Space

Fig.3: an array of frames in Personal Space with the frame alpha ( $\mathrm{f} \alpha$ ) being the most recently created frame

\section{Clusters and Sectors}

A cluster is a grouping of coherent QF's within a frame and generally across a few frames in personal space that contains a collection of all the QF's, which belong to a single stimulus. Every object one can label, every sound one can meaningfully perceive i.e. every perception of a stimulus in the classical sense, is the result of the creation of a cluster. Sectors in the QFT refer to the sequence of frames aligned one after another that are created by exerting focused attention on certain aspects of the environment and have the potential to be perceived as coherent groups as they share several interconnected LDU's of SI and LA. The coherence of the sector is precipitated by the QF's that it holds within its frames as they share a relationship or relatedness between themselves. A sector is thus an area in personal space that is a coherent rather than a random collection of information. Sectors are longer than clusters. A sector would generally contain several clusters. If you take decide a walk of your home all the frames that you create from the time you begin your walk till the end would be termed as a sector and all the individual objects you encounter in your home throughout your walk would be clusters. 


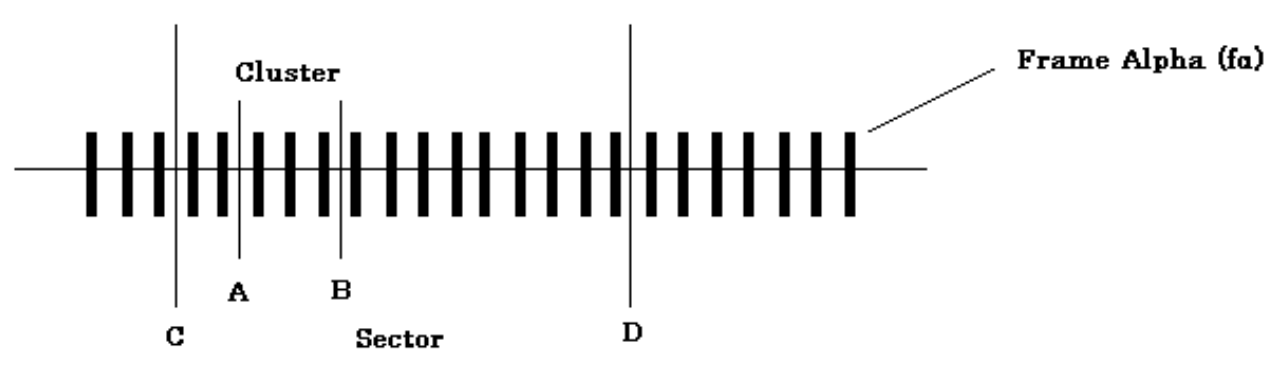

Array of Frames in Personal Space

Fig.4: A Cluster within a Sector in Personal Space

\section{Functional Components of the Theory}

The understanding of the functional components is imperative for the comprehension and appreciation of the implications that the theory envisions. Functional components include vibrations, two way Influence, cross modal quanta factor vibration, resonance wave of personal space, guided assimilation based awareness, the motor component and the sector command.

\section{Vibration}

Quanta factors are proposed to be in a constant state of vibration. Each half of an individual QF namely the LDU of SI and the LDU of LA have a separate pattern of oscillation and the QF that they make up intern has a unique patter of vibration of its own. Just as different wavelengths of light give us different colors, the QFT envisions vibrations that oscillate in a unique manner for each individual Least Divisible Unit of Sensory Information and Latent Affect that ever has or can ever exist. No two different LDU of SI or LA have a similar oscillation pattern. However most crucially, two perfectly similar LDU of SI or LA shall always have the same oscillation pattern.

\section{Two Way Influence}

When any one half of a QF stored in personal space vibrates the other half of that QF also vibrates. If the LDU of SI of a QF vibrates then the LDU of LA of that QF shall also vibrate.

\section{Cross Modal Quanta Factor Vibration}

When one quanta factor from a given frame vibrates then due to the similarity of the latent affect among all the quanta factors in that frame all the quanta factors in that frame begin to vibrate. When two way influence and the cross modal quanta factor vibration are read together it is understood that when any one half of a QF within a frame vibrates then an inevitable consequence is that the entire frame begins to vibrate.

\section{Resonance}

Whenever new QF's are created in the fa by the merging of the LDU's of SI and LA, these QF's influence exactly similar LDU's of SI and LA that are part of previously stored QF's to oscillate faster and due to the process of two way influence and cross modal quanta factor vibration the entire frame in which those similar LDU's of SI and LA are stored vibrates robustly and copies of all the vibrations in the frame are expelled out of the frame into the personal space. This influence of newly created QF's on previously created QF's in the QFT is termed as resonance.

\section{The Wave of Personal Space}

When a frame vibrates as a whole it is proposed by the QFT that copies of the vibrations of each LDU of SI and LA contained in that frame are released and are expelled into the personal space. These copies of LDU's of SI and LA move both forwards and backwards in personal space. When they move forward they 
are moving towards the frame that is currently being created i.e. the frame alpha, fa. It must be clearly understood that a facreates resonance and a wave of personal space is generated, now information in the wave is available for merging in the currently being created frame i.e. the next fa. So the frame that generates the wave is $f \alpha-1$, and the new fo incurs its effects.

\section{SET A Information and SET B Information}

At the fa two types of information are present to be merged and stored as QF's. One is the information coming from the current environment and the second is the information carried by the wave of personal space. Both the current environment and the wave of personal space present SI and LA at the fo. Though LA can be generated from personal space only but it has been differentiated based on its point of origin, i.e. is the LA generated due to something in the current environment or is it due to the information from personal space. Thus information in the QFT is divided into four different categories sensory information current environment SICE, latent affect current environment LACE, sensory information personal space SIPS and latent affect personal space LAPS.

\section{The Affect Line}

The Affect Line is a hypothetical conceptualization of the entire range of affects experienced by individuals. Affects are proposed to be placed next to each other on the affect line. However they are not envisioned to be placed on the affect line as clearly distinct entities. The conceptualization of latent affects in the QFT is analogous to the conceptualization of the electromagnetic spectrum. Just as how frequencies of electromagnetic radiation move gradually from one to another so are latent affects proposed to change from one affect to another.

Each independent frequency is to be imagined as an independent latent affect. Visually as we see the spectrum as one hue of color changes into another there is an area between two distinct colors that is neither of the two colors. Say for example from green to yellow. As we visibly see the green change into yellow it's a gradual change where some part of the spectrum is nor exactly green nor is it exactly yellow. As we move from left to right the color gradually becomes lesser and lesser a characteristic of the former green and more and more of the latter yellow. A similar conceptualization is proposed for the latent affects. If we were to imagine that the entire range of 'fear' as general affect is spread over 20 fractionally distinct latent affects, then just like the colors on the electromagnetic spectrum there are proposed to be two other general affects with their range of fractionally distinct latent affects on either side of the general affect 'fear'. So it must be imagined that the first five and the last five fractionally distinct latent affects of the affect fear, also have to some degree the characteristic of the affects on either side of fear, (whichever affects they might be) and the middle few are to a high degree characteristic of the affect we have labeled fear. The term general affect however does not suggest that 'general affects' in the QFT means what are commonly understood as emotions like fear, joy, disgust etc.

\section{Degrees of Freedom of Affective Categorization}

This refers to the critical number of LDU's of Latent Affects required to be present in a cluster to allow the individual to affectively experience the cluster as one specific general affect. As affects are conceptualized as a range, and as explained like the electromagnetic spectrum at the merging areas between two specific general affects some latent affects would have in varying degrees the characteristics of both the general affects. Whenever an individual encounters a stimulus and creates a cluster that is made up of LDU's of LA's that happen to fall from these areas or when any cluster is made up of LDU's of LA's that are part of characteristically different general affects, there would be affective confusion as to the nature of the affect experienced. In such situations the cluster shall be given the general affective quality of (or be experienced as belonging to), the affect that has the highest number of LDU's of latent affects represented in that cluster. This type of envisioning of latent affects is integral to the QFT formulation of concepts and categorization. 


\section{The Point of Equanimity (PoE)}

The Point of Equanimity (PoE) in the QFT is a hypothesized latent affect that is characterized as a sensation of a high degree of satiety. It is akin to a feeling of being in a state of need fulfillment. It is frequently achieved and lost. Not to be confused with nirvana or similar states of consciousness, it is a feeling of satisfaction. If you are at the point of equanimity it simply means that the latent affect that is merging with incoming LDU's of SI at this moment does not demand you to perform a physical or mental action. Thus it is a feeling of being content.

It must be clearly understood that the point of equanimity is an hypothesized point that is part of a larger affective range. In essence the hypothesized affect that is labeled as being at equanimity would itself be a range as all the other affects are hypothesized to be. As in when individuals are merging latent affects that fall within the range of equanimity they are experiencing varying levels of equanimity as is the case with the range of other affects.

\section{Voluntary and Subjective Character of the Point or Range of Equanimity}

The latent affects that are to be considered as falling under the range of equanimity for any individual varies at any given time and can also be absolutely any affect. On account of voluntary or involuntary changes in the latent affects within the individual the point of equanimity shifts its position on the affect line. A voluntary shift occurs when an individual willfully changes his needs or goals and an involuntary change occurs when an occurrence in the environment forces the individual to move away from the point of equanimity or changes the affect within the individual that has shifted him from his original position on the affect line.

Thus point of equanimity is subjective and changing. What might characterize as equanimity to one person may not be so for another. What might be the point of equanimity at a given point in time may not be so at another point in time for the same person.

With respect to their effect on the movement of the current affect in relation to the point of equanimity, affects are categorized as positive or negative affects. If the latest generated affect post the setting of the point of equanimity is one that is closer to the point of equanimity than the affect that was being experienced previously then the current affect is said to be a positive affect. And if the generated affect is one that has moved farther away from the point of equanimity than the affect that was being experienced previously then the current affect is said to be a negative affect. The positive and negative labels do not refer to any good or bad nature of the affects, (e.g. it does not suggest that happy is positive and sad is negative), it only means that all the affects that characterize a shift closer to the point of equanimity induce a positive movement on the affect line and all the affects that characterize a shift away from the point of equanimity induce a negative movement on the affect line.

The proposal of the QFT regarding the PoE must be clearly understood as it has implications for almost all behaviors and cognitive processes in the quanta factor theoretical frame work. When you are trying to quench your thirst, satiate your hunger, achieve a behavioral or mental goal (e.g. going to the market and buying stuff or trying to remember something or make decisions) in all these tasks you are attempting to move your current affect towards the PoE. Individuals are thus constantly voluntarily or involuntarily shifting away from the PoE and are attempting to move towards the PoE.

\section{The Motor Component (MC)}

Reflexive and voluntary actions and behaviors of human beings find a place in the QFT in the form of the "motor component". The motor component $(\mathrm{mc})$ is seen as an urge to display an action or behavior, reflexively or voluntarily. This urge is a response to an individual LDU of LA or a group of latent affects.

This is an important proposal in the QFT regarding the motor component that bases the functioning of the motor component on the latent affect. In traditional terms the QFT proposes that all human actions and behaviors whether they might be reflexes, or voluntary and planned actions, actions involving procedural knowledge i.e. all motor behaviors are proposed to occur as a response to latent affects.

Any movement of the human organism is seen to occur due to the following reasons, 
1. Reflexively when an external or internal stimulus has caused a shift in the latent affect changing one's current affect away from the point of equanimity, then a motor component might be activated to cause certain physical changes in the internal or external environment that attempts to bring the current affect to the point of equanimity,

2. Voluntarily during planned activity, these long coordinated collection of movements are also proposed to be activated and coordinated as directed by continuous feedback of the effectiveness of the activated motor component assessed by the effect it has on the latent affect.

The QFT divides the MC into four types: the motor component inertia (MCI), the motor component rapid (MCR), the motor component 1 (MC1), and the motor component 2 (MC2). The MCI is associated with an LA that does not demand physical action i.e. when you are simply resting on your bed. The mcr is activated by an independent LDU of LA. This type of motor component can never be voluntarily activated. The mc1 are activated by a group of LA's and are reflexive and involuntary actions/behaviors. The MC1 is a collection of a group of MCRs and MCIs. If at the corner of your eye you see something move towards you and you instinctively flinch, this flinching action is what would be the mc1, and all the subtle muscle movements that you made (unconsciously) would be the activation of the MCR, any physical activity of a longer duration is accomplished by the activation of the MC2. In the MC2 action/behavior is maintained by the individual by holding on to preferred affects usually in order to accomplish goals. MC2s are the longest coordinated motor components envisioned in the QFT.

Thus every human voluntary and involuntary movement is linked to the latent affect. If you were to walk from point $\mathrm{A}$ to $\mathrm{B}$ then every twitch of the muscles required to produce the walking motion would be the MCRs, the entire physical movement from A to B would be the completion of the MC2, and once you reach $B$ as you would not require to do any physical movement you would be utilizing the MCIs. If you were to slip during your movement from A to B the all the actions you would perform to stabilize yourself would be utilization of a MC1. One could say the function of the physical actions provide the difference in the labels given to the motor component.

Reflexive and short or long goal oriented physical behavior is thus in the QFT proposed to be due to the use of the MC based on the voluntarily and involuntarily generated latent affect. And all physical behavior i.e. the use of any $\mathrm{mc}$ is by conscious volition or automatically attempting to move the current LA towards the point of equanimity.

The important concept that allows goal oriented behavior is that of the sector command.

\section{Sector Command}

Sector commands are free will affective demand articulations that shall entail the creation of both a goal and a code that aids in the achievement of that goal. This goal in the form of the achievement and/or maintenance of a particular desired affect shall involve the use of the motor component to achieve the set goal.

If you are sitting on your couch at home and feel thirsty, the decision you make to go drink water is the generation of a sector command. While this sector command is on, you shall be urged by this sector command to focus on the clusters in the environment that are integral to the successful completion of the activity that is the quenching of your thirst. The main goal of the sector command is to shift your latent affect towards the point of equanimity (which is hoped to be achieved once your thirst is quenched) and to achieve this goal safely. This is envisioned as occurring for long term goals as well. If you have set a sector command to do well on an annual exam then all activities that would be considered as helping you to achieve that goal would be in QFT terms creating positive affective shifts, be it putting in long hours of study, sleeping well, attending lectures etc. And all activities that take you away from your goal create negative shifts on the affect line, this mechanism utilizes the AMSG to gather feedback of your actions and helps maintain positive shifts on the affect line to achieve the sector command. 


\section{Environment (Stimuli) Organism - Status Quo (ESO-SQ), and the Affect Motor Sensory Guidance Mechanism (AMSG)}

When organisms (individuals), physically interact with their environment they risk harm. The QFT envisions for the maintenance of the organism's safety an adaptation and a mechanism that work together constantly to keep an organism safe. It is envisioned in the QFT that they work towards the maintenance of the individual's safety during every twitch, reflex and goal directed movement. The specific adaptation envisioned to maintain an organisms safety in the QFT is the Environment (stimuli) Organism - Status Quo (ESO-SQ), and the mechanism envisioned to achieve and maintain the ESO-SQ is the Affect - Motor Sensory Guidance Mechanism (AMSG).

The environment (stimuli) - Organism status quo is a proposed primal perceptual adjustment adaptation envisioned in the QFT that allows an organism to attempt to constantly maintain a status quo with the varied array of stimuli one finds himself surrounded by. The goal of this proposed perceptual adaptation is to attempt to provide an extremely rapid, transitory but an informative affordance to an organism vis-à-vis the stimuli that one is encountering i.e. one's current environment. The affordance provided by this status quo is in its potential to help the organism to stay a step ahead of the stimuli present in its environment and to avoid hurt or confusion and provide an informative blue print for any motor behavior be it reflexive or planned behavior.

The evaluation of the ESO-SQ is done by assessing the shift in the LA within the individual in response to the stimuli in the environment. The ESO-SQ is maintained by the maintenance of the LA at a desired place on the affect line usually while performing an activity or while bringing back the current LA to a desired point on the affect line.

The method of maintaining or bringing back the latent affect to the desired type of latent affect or to the point of equanimity is by the activation of the motor component. The usage of the motor component to get the affect towards the point of equanimity while taking into account the sensory information whenever available in the current environment is termed as the Affect Motor Sensory Guidance (AMSG). It must be understood that this maintenance of the ESO-SQ and the AMSG is proposed to be continuously going on at each frame. This process primarily maintains the ESO-SQ and keeps the organism safe but is also what allows the successful completion of goals, guiding and planning of actions and decision making. In simple terms when you want to accomplish something the affect that would be achieved when you accomplish that task would be set as the point of equanimity. Now every action (utilized motor component linked to latent affect) that you do post the setting of the PoE is accessed via the affect that it generates in term of whether it is a positive or a negative affect. Based on this feedback the subsequent actions would be materialized by the generation of the latent affects that continue to take you towards the PoE or those that rectify the negative shift created by the previous actions. If you are thirsty then the affect you would experience once you quench your thirst would be set as the PoE. As a consequence you would generate latent affects that in turn generate motor components that allow you to reach the set PoE. Taking a step towards the water bottle would be generating MCs that take your current affect towards the PoE. Throughout this process the ESO-SQ is always on. If you see something threatening on your way to the bottle then the PoE may change requiring different mc's that are generated by latent affect that take you to safety i.e. to the now set PoE.

This is a simple analogical explanation however this working of the ESO-SQ and the AMSG mechanism is proposed to be integral generally to human survival, and specifically to all cognitive processes that involve the accomplishment of goals.

\section{Guided Assimilation Based Awareness (GABA)}

Though all constructs and functions proposed and explained in the QFT are significantly interconnected it could be suggested that the Guided Assimilation Based Awareness is by far the most pervasive by reason of its implications. Its most important function is in essence to make us experience our individual version of reality. It is the GABA that dictates several of our cognitive processes by means of what the QFT refers to as the three rules of conscious perception. During the creation of the fa, both SET A and B information are competing to be merged. Not all the information gets transformed into QF's and gets merged in the fo. The QFT lays down three rules of how and how much information shall get merged in the fa from both 
SET A and B. For the purpose of this review I shall only state rule one and two and elaborate only on rule three.

- Rule 1: LDU's of SI and LA expelled from one frame from personal space shall, (if selected based on rule 3), have the potential to be merged in only one frame i.e. the current fa.

- Rule 2: The current frame can assimilate LDU's of SI and LA expelled by multiple frames.

- Rule 3: The assimilation of LDU's of SI and LA from the wave of personal space into the fo is a function of the intensity LDU of LA and the similarity between the LDU's of SI.

Now the QFT has stated that there can be only one LDU of LA as the half of all the LDU's of SI in one frame, i.e. all the QF's in one frame have the same LDU of LA as one half of them. The QFT states that between the two LDU's of LA competing at f $\alpha$, (LACE and LAPS) that LDU of LA which is of higher intensity is selected. Once the LDU of LA of the f $\alpha$ is decided all the QF's present in the wave that have this LDU of LA as one half of them have a high probability to be assimilated in the fa. But only those LDU's of SI from personal space that are similar to the LDU's of SI currently encountered in the environment shall have a high probability to be assimilated and this process shall give you a conscious awareness.

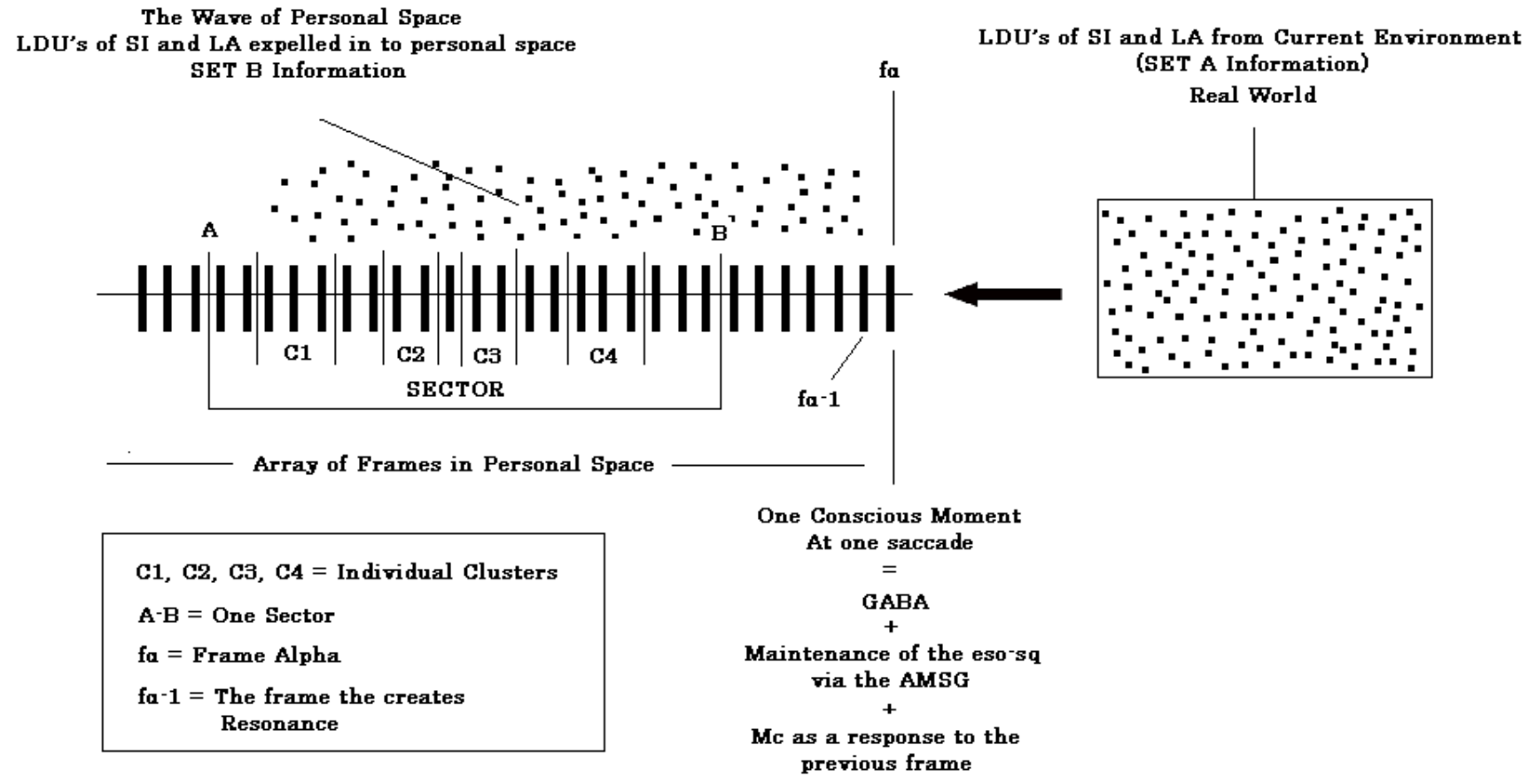

Fig.5: An outline of the creation of a f $\alpha$ i.e. one conscious moment at one saccade.

\section{A few implications of the QFT}

The structural and functional concepts provided by the QFT are proposed to be sufficient to provide a universal model of human behavior and mental processes. The QFT conceptualizations of a few of the cognitive processes have been elaborated in the book "The Quanta Factor Theory of the Human Mind" but for the purpose of this chapter I shall elucidate in simple terms how the concepts in the QFT translate into models of a few cognitive processes. These implications and proposals have to be subjected to empirical inquiry in the future.

Consciousness is the creation of frames. The time of frame creation is currently set by the QFT as one frame per saccade. The influence of the AMSG with the use of the mc, in its task of maintaining the eso-sq even between two frames entails that these processes are working even between saccades. There is a constant resonance, creation of the wave of personal space and merging of information in the f $\alpha$ based on the GABA. Thus consciousness is a continuous process. 
In simple terms perception is the creation of clusters in personal space. When selective attention is applied on a stimulus conscious perception of that stimulus is said to occur and in QFT terms a p-clusters of that stimulus is created. The QFT differentiates between an a-cluster and a p-cluster. A p-cluster of a stimulus holds within itself all the LDU's of information that allow the individual to physically experience, know and maintain the eso-sq with that stimulus. An a-cluster is one which holds only that information that allows a physical experience of that stimulus.

Selective attention is proposed to be the result of the intensity of the latent affect criteria part of the rule 3 of the GABA. When presented with an array of stimuli then everything are senses can detect is broken down into their LDU's and are merged with the LA and QF's are formed and stored in frames. However among these stimuli there shall be one stimulus that generates the strongest LA and consequently only the cluster of that stimulus can be consciously perceived ie a p-cluster of that stimulus is formed. All the other clusters being formed at the same time are part of our awareness but not consciously perceived and are termed as a-clusters. The bottleneck or flashlight in the QFT is based on the intensity of the LA.

Memory is the remerging in the present of clusters and sectors that have been previously created and stored in personal space. These clusters and sectors remerge as directed by the rules of the GABA. Now as GABA is influenced by the current environment no remerging can be exact. Thus memories would probabilistically include information from both SET A and SET B. The GABA process can cause current and past sensory and affective information to remerge and create slightly or significantly different new clusters or clusters within the sector being remerged. The QFT thus provides its conceptualization for the reconstructive nature of memory.

Working Memory (WM) is the constant and continuous functioning of the GABA, the sector command, the ESO-SQ and the AMSG mechanism. Though WM according to the QFT needs much more explaining, in the most basic terms it is proposed that any functional consequence that is proposed by the components of the WM model can be explained as a consequence of the components of the QFT.

Categorization is linked to the degrees of affective categorization. The affective experience of any stimulus via the LDU's of LA dictates which category of objects that stimulus falls within. According to the QFT we experience or categorize a stimulus as a being part a specific category when the LDU's of LA that are part of the cluster that you have just created to experience that stimulus are part of a general affect that pertains to that specific category of objects. This general affect pertaining to one category of objects is subjective. A general affect that one individual have in place for a category of say 'dogs' can differ from that of another person.

Decision making and problem solving are based on the setting of the point of equanimity. With decision making an individual sets a PoE and creates sectors in SET B and assesses which sequence or selection of sectors gets him to the PoE or gets him to the PoE fastest. In problem solving the affect achieved post the solution of the problem is the PoE. The individual undertakes actions by utilizing the mc and assess the effectiveness of each action based on the affective shift that action has caused in either positive or negative shift on the affect line. Based on this feedback the individual either continues with the sequence of action or undertakes an alternate course of action and continues this process till the PoE is achieved.

The QFT conceptualizes language as an outcome of the AMSG mechanisms attempt to maintain the esosq by considering language as an auditory stimulus in the environment and proposes language to be a reflex. In the QFT language comprehension is a secondary byproduct of the primary requirement of maintaining the ESO-SQ. The understanding of words as clusters and sentences and paragraphs experienced as sectors and especially the linking the non comprehension of sentences and speech as a threat to the ESO-SQ promises to open up a tremendous amount of inquiry and conceptualization.

Dreams are suggested to be the merging of LDU's of SI and LA (information) from SET B based on the rule 3 of the GABA. The QFT suggests that while asleep the LA from the current environment is so attenuated that we experience SET B merging as real in the same way as we experience SET A information when we are awake even though SET B information is still being merged. When we are awake we need to maintain the ESO-SQ with the stimuli in the current environment and thus they produce a more intense LA (automatically) and we experience SET A clusters more intensely. The opposite becomes true when we are asleep. 
In the QFT one could say that there are no observer independent categories of objects. No reality is devoid of human observation and each observation is a separate subjective reality. Perception and experience are due to the formation of clusters and sectors in personal space. Based on volition and probability due to the rule 3 of the GABA clusters and sectors from SET A or B could both be experienced as real at any given time. This conceptualization of the mind and reality can be explored to formulize a QFT conceptualization of mental disorders that involve altered experiences and drug induced altered states of mind. The reality of any real world stimulus in the QFT is never only sensory information, it also includes affective component. As such in the QFT no two individual experience the same object in the exact same way.

Though I have called the most integral component of the theory as a 'quanta' factor I have not proposed the theory as a quantum model of mind or consciousness. However several of the quantum models of consciousness involve concepts that are similar to those in the QFT. Resonance and the resulting action of two similar but separate quanta factors suggest entanglement as seen between particles. The presence of SET A and B information at the $\mathrm{f} \alpha$ and the subsequent probabilistic selection and creation of quanta factors and the creation of a frame could be regarded as a quantum collapse. Remember that the QFT is a model, however what real physical objects whether neuronal, sub neuronal or even quantum particular material quanta factors are models of, is itself I believe is the biggest matter of research and debate.

\section{CONCLUSIONS}

The proposal of the QFT is in a way a top down endeavor. The attempt is to first present an acceptable model of the mind, consciousness, cognition and human behaviors and subsequently look for empirical, biological, neural, genetic and metaphysical correlates. The QFT could be considered as a model of the mind that elaborates how moments of consciousness are created and how we experience a continuous consciousness. The same model also provides concepts and mechanisms for human mental processes and behaviors. The latent aspiration of the theory is that by establishing the veracity of the explanations of separate cognitive faculties and behaviors via one single model the acceptance of the theory as a theory of consciousness as a whole becomes plausible.

\section{RECOMMENDED REFERENCES AND READING}

1. Baddeley AD, Hitch G. Working memory. In GH Bower (Ed.), The psychology of learning and motivation: Advances in research and theory (Vol. 8, pp. 47-89). New York: Academic Press ; 1974.

2. Bayne T, Cleeremans A, Wilken P (eds.). The Oxford Companion to Consciousness. Oxford University Press : Oxford, UK ; 2009.

3. Kathleen M, Galotti G. Cognitive psychology in and out of the laboratory (3rd ed). Belmont, Calif Wadsworth ; 2003.

4. Kimble GA, Garmezy N, Zigler E. Principles of General Psychology (6th ed). New Delhi: Wiley Eastern Limited, 1985.

5. Lahey BB. Psychology: An Introduction, 6th Edition. McGraw Hill, Boston, MA, 1998.

6. Nisbett RE, Ross L. Human inference: Strategies and shortcomings of social judgment. Englewood Cliffs, N.J: Prentice-Hall ; 1980.

7. Pillai R. The Quanta Factor Theory of the Human Mind. Mumbai. Rohan Pillai ; 2015.

8. Tarlaci S. Why we need quantum physics for cognitive neuroscience. NeuroQuantology 2010;8(1):66-76.

9. Bohm D. A new theory of the relationship of mind and matter. Philosoph Psychol 1990;3(2-3):271-86.

10. Thibault P. Brain, mind and the signifying body: An ecosocial semiotic theory. A\&C Black; 2004.

11. McFadden J. The conscious electromagnetic information (cemi) field theory: the hard problem made easy?. J Conscious Stud 2002;9(8):45-60.

12. de Maré P, Schöllberger R. A theory of mind. Group Analysis 2006;39(1):65-71.

13. Korf J. Quantum and multidimensional explanations in a neurobiological context of mind. The Neuroscientist. 2014 Jul 21:1073858414542250.

14. Heisenberg W. The physical principles of the quantum theory. Courier Corporation; 2013.

15. Halpern P. Einstein's Dice and Schr dinger's Cat: How Two Great Minds Battled Quantum Randomness to Create a Unified Theory of Physics. Basic Books; 2015. 
16. Ward BK. Is There a Link Between Quantum Mechanics and Consciousness?. InBrain, Mind and Consciousness in the History of Neuroscience (pp. 273-302). Springer Netherlands ; 2014.

17. Rapparini R. The Mind of the Beholder. Quantum 2015;6(1):181-8.

18. Jaroszkiewicz G. Images of Time: Mind, Science, Reality. Oxford University Press; 2016.

19. Lancaster T, Blundell SJ. Quantum field theory for the gifted amateur. OUP Oxford; 2014.

20. Hawking S. Quanta. Physics World. 2015 May;28(5):3.

Acknowledgements - Nil

Source of Funding - Nil

Conflict of Interest - Nil 